\title{
Impacto de un curso propedéutico en las competencias matemáticas de estudiantes de nuevo ingreso de licenciatura del TecMM campus Puerto Vallarta
}

Impact of a propaedeutic course on mathematical competencies in new college students of the TecMM Puerto Vallarta Campus

Impacto de um curso preparatório nas competências matemáticas de novos alunos de graduação no campus da TecMM em Puerto Vallarta

Carlos Miguel Amador-Ortiz Instituto Tecnológico José Mario Molina Pasquel y Henríquez, Campus Puerto Vallarta,

México

carlos.amador@vallarta.tecmm.edu.mx https://orcid.org/0000-0001-6654-8448

María Luisa Torres-Isiordia Instituto Tecnológico José Mario Molina Pasquel y Henríquez, Campus Puerto Vallarta, México maria.torrez@vallarta.tecmm.edu.mx https://orcid.org/0000-0001-5125-8018

Leticia Velarde Peña

Instituto Tecnológico José Mario Molina Pasquel y Henríquez, Campus Puerto Vallarta, México carlos.amador@vallarta.tecmm.edu.mx https://orcid.org/0000-0003-1096-1035 


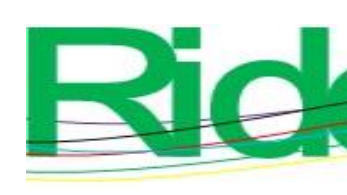

Revista Iberoamericana para la
Investigación y el Desarrollo Educativo
ISSN $2007-7467$

\section{Resumen}

Las universidades imparten un componente formativo de matemáticas, el cual puede ser básico o complejo dependiendo del área de estudios (las ingenierías llevan por lo general una formación más enfocada al desarrollo de estas competencias). Una problemática que se presenta en las instituciones de educación superior es el alto índice de reprobación en matemáticas (con implicaciones en la deserción), lo cual se debe a que los estudiantes ingresan sin contar con las competencias esperadas de los niveles formativos previos. Por eso, el objetivo del presente trabajo fue determinar el impacto de un curso remedial diseñado por la coordinación de ciencias básicas del Instituto José Mario Molina, Pasquel y Henríquez, campus Puerto Vallarta (TecMM), en la formación de competencias matemáticas en estudiantes de nuevo ingreso. Para ello, se utilizó un cuestionario diagnóstico con temas de aritmética y álgebra, el cuál se aplicó mediante un formulario web antes y después del curso remedial a 134 estudiantes. Asimismo, se utilizó una prueba Z dependiente de muestras pareadas para determinar el nivel de significancia en la diferencia de las dos mediciones. Los resultados fueron sometidos a una prueba de hipótesis estadística mediante la cual se demostró que la implementación del curso remedial fue funcional. Se concluye, por tanto, que esta es una estrategia útil de nivelación para estudiantes de nuevo ingreso.

Palabras clave: competencias matemáticas, curso propedéutico, educación superior, estrategia, reprobación.

\section{Abstract}

Universities teach a training component in mathematics, which can be from a basic level to a more complex one, depending on the area of study (engineering generally has training more focused on the development of these competencies), a problem that occurs in the college institutions is the high rate of failure in mathematics (with implications for dropout), because students enter without having the skills expected from previous educational levels. The purpose of this work was to determine the impact of a remedial course designed by the basic sciences coordination of the José Mario Molina, Pasquel y Henríquez Institute, Puerto Vallarta campus (TecMM) in the formation of mathematical competencies in new students. To develop the study it was used a diagnostic test with arithmetic and algebra topics which was applied through a Web form before and after the remedial course to 134 students, and a dependent $\mathrm{Z}$ test for paired samples was used to determine the level of significance in the 


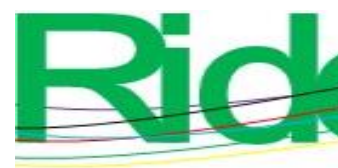

Revista Iberoamericana para la
Investigación y el Desarrollo Educativo
ISSN $2007-7467$

difference of the two measurements. The results show a significant improvement after the implementation of the course, concluding that this is a useful leveling strategy for new students.

Keywords: mathematical competences, propaedeutic course, college education, strategy, failure.

\section{Resumo}

As universidades ensinam uma componente de formação em matemática, que pode ser básica ou complexa dependendo da área de estudo (a engenharia geralmente requer uma formação mais focada no desenvolvimento destas competências). Um problema que ocorre nas instituições de ensino superior é a elevada taxa de reprovação em matemática (com implicações para a evasão), que se deve ao facto de os alunos ingressarem sem possuir as competências esperadas dos níveis de formação anteriores. Portanto, o objetivo deste trabalho foi determinar o impacto de um curso corretivo elaborado pela coordenação de ciências básicas do Instituto José Mario Molina, Pasquel e Henríquez, campus Puerto Vallarta (TecMM), na formação de competências matemáticas nos alunos novamente entrada. Para isso, foi utilizado um questionário diagnóstico com tópicos de aritmética e álgebra, o qual foi aplicado por meio de um formulário web antes e depois do curso de reforço a 134 alunos. Da mesma forma, um teste $\mathrm{Z}$ dependente de amostras emparelhadas foi usado para determinar o nível de significância na diferença das duas medidas. Os resultados foram submetidos a um teste de hipótese estatística através do qual foi demonstrado que a implementação do curso corretivo foi funcional. Conclui-se, portanto, que esta é uma estratégia de nivelamento útil para novos alunos.

Palavras-chave: competências matemáticas, curso preparatório, ensino superior, estratégia, fracasso.

Fecha Recepción: Mayo 2021

Fecha Aceptación: Noviembre 2021 


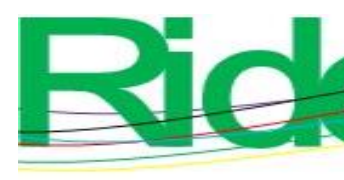

Revista Iberoamericana para la Investigación y el Desarrollo Educativo ISSN $2007-7467$

\section{Introducción}

En México, la educación media superior registra rezagos significativos en diversos indicadores, tales como problemas de baja cobertura y eficiencia terminal, así como altos índices de reprobación y deserción (Castro, Piñon y Avilés, 2015). Estas problemáticas tienen un impacto en los alumnos que ingresan a nivel superior (Gil y Pérez, 2013), quienes por lo general presentan deficiencias en algunas competencias básicas, entre ellas la matemática. Esta competencia — según García-Oliveros et al., (2020) — puede ser definida como "la disposición perspicaz para actuar en respuesta a los desafíos matemáticos de una situación dada y en la identificación de las capacidades que la componen: razonamiento, representación, comunicación, simbolismo-forma, resolución de problemas y modelamiento" (p. 252).

En México, la Secretaría de Educación Pública (SEP) aplica la evaluación estandarizada PLANEA a estudiantes de educación media superior que cursan su último semestre para conocer el nivel de los aprendizajes obtenidos en lenguaje y matemáticas, los cuales se categorizan en cuatro niveles: el nivel I corresponde a conocimientos insuficientes; el nivel II a conocimientos elementales; el nivel III a conocimientos satisfactorios y el nivel IV a conocimientos sobresalientes.

En 2017, los resultados de Jalisco en matemáticas fueron los siguientes: $54.91 \%$ en nivel I, $26.54 \%$ en II, $12.4 \%$ en III y $6.15 \%$ en IV. En cambio, a nivel nacional se ubicaron del siguiente modo: $66.2 \%$ en nivel I, $23.3 \%$ en II, $8 \%$ en III y $2.5 \%$ en IV (SEP, 2017).

Con esos resultados en las pruebas de PLANEA (deficiencias principalmente en matemáticas), se identifica la antesala de una problemática que se presenta en educación superior; es decir, un alto porcentaje de alumnos de nuevo ingreso no poseen las competencias básicas en matemáticas y en razonamiento lógico, lo que les dificulta culminar de forma exitosa sus estudios universitarios. Sobre esta realidad, Zámková, Prokop y Stolín (2016) mencionan que las universidades están aceptando a estudiantes con deficiencias debido a que hay pocos candidatos elegibles. Además, en un estudio con universitarios se encontró que ellos consideran regular su manejo de conocimientos previos relevantes (Martínez et al., 1998). La falta de conocimientos previos esperados en las universidades es preocupante por el efecto que se puede generar a futuro en la calidad formativa de los recursos humanos (Gaete y Jiménez, 2011). 


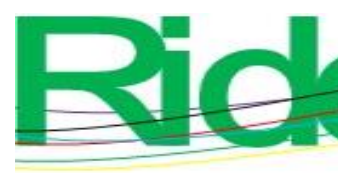

Revista Iberoamericana para la
Investigación y el Desarrollo Educativo
ISSN $2007-7467$

La indagación de las causas de reprobación en matemáticas da cuenta de factores de diversa naturaleza. Al respecto, Martínez et al. (1998) categorizan los factores en tres: sociales, institucionales e individuales. En específico, los autores encontraron que competencias previas, el desempeño docente y estudiantil, así como el aspecto emocional del alumno, su vocación y la infraestructura institucional pueden incidir en la reprobación en matemáticas.

Autores como Romero-Bojórquez, Utrilla-Quiroz y Utrilla-Quiroz (2014) explican que "existen referentes experienciales, que provocan predisposiciones o actitudes que afectan la incorporación del alumno al proceso de aprendizaje, y en su aprovechamiento escolar o reprobación” (p. 293). Otros, además, opinan que la actitud de una persona influye en su aprendizaje, pues dicho factor incide en lo cognoscitivo, afectivo y conductual. Sobre esto, Vila y Rubio (2016) mencionan que "se aprende mejor aquello que produce mayor agrado, y una educación adecuada puede mejorar las actitudes de los estudiantes ante una materia determinada" (p. 135). Asimismo, Shena, Mielea y Vasilyeva (2016), sobre la persistencia de los estudiantes para el aprendizaje de las matemáticas, concluyen que "la mentalidad académica de los estudiantes influye en su interpretación de sus experiencias de resolución de problemas matemáticos, así como su persistencia en la resolución de problemas subsiguientes" (p. 51).

Cabe mencionar que la capacidad cognitiva y la habilidad de atención se encuentran relacionadas con el rendimiento académico; además, el uso de estrategias avanzadas está asociado con la resolución de problemas matemáticos. La motivación, en consecuencia, desempeña un papel importante. Al respecto, Petriz et al. (2010) utilizaron un instrumento para evaluar contenidos y otro para medir motivación, agrado, ansiedad, utilidad y confianza. Sus conclusiones indican que "los estudiantes que manifestaron una mayor motivación resolvieron los problemas de mayor complejidad" (p. 1238). También Vargas y Montero (2016) encontraron que las actitudes negativas hacia las matemáticas se relacionan con un efecto directo (negativo) en el rendimiento académico.

Sumado a todo lo anterior, vale resaltar que cada estudiante tiene una determinada forma de aprender, de ahí que requiera estrategias didácticas específicas. Por tanto, resulta de vital importancia la planificación docente para generar respuestas emocionales positivas mediante metodologías de enseñanza que faciliten los procesos de aprendizaje (Yánez y Nevárez, 2018). 


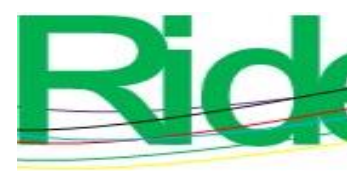

Revista Iberoamericana para la
Investigación y el Desarrollo Educativo
ISSN $2007-7467$

De acuerdo con Posso (2005), “el proceso de enseñanza debe adecuarse al nivel de razonamiento del alumno" (p. 172), lo que se dificulta en las aulas de educación superior en el área de matemáticas debido a las deficiencias en los conocimientos previos (Vaca Haro y Estévez, 2017).

Por tal motivo, el presente trabajo tuvo como propósito determinar el impacto de un curso remedial diseñado por la coordinación de ciencias básicas del Instituto José Mario Molina, Pasquel y Henríquez, campus Puerto Vallarta (TecMM), para la formación de competencias matemáticas en estudiantes de nuevo ingreso.

\section{Materiales y métodos}

Esta investigación aplicada tuvo un enfoque cuantitativo, con alcance correlacional, mediante un diseño cuasiexperimental con preprueba y posprueba. Para ello, se usó un método inductivo. La unidad de análisis fue el TecMM, mientras que la población objetivo fueron los estudiantes de nuevo ingreso. Se tomó como muestra a los alumnos que aplicaron el examen diagnóstico y un examen de cierre de curso propedéutico.

Las variables involucradas fueron curso propedéutico (variable independiente) y competencias matemáticas (variable dependiente).

\section{Instrumento de recolección de información y aspectos metodológicos}

El instrumento utilizado fue un cuestionario de problemas matemáticos que se aplicó en formato digital mediante un formulario web como preprueba (antes del curso propedéutico) y posteriormente como posprueba (después del curso propedéutico) para identificar las diferencias entre los dos resultados. Este instrumento estuvo constituido por dos dimensiones: aritmética y álgebra.

Para la selección de los temas del cuestionario y los contenidos del curso propedéutico se consideraron las competencias esperadas de nivel media superior, y los prerrequisitos para las materias de nivel licenciatura del TecMM, campus Puerto Vallarta. Las competencias previas que se le solicitan al alumno para poder cursar su primera asignatura de matemáticas en el nivel superior (considerando las diferentes carreras y asignaturas) son operaciones aritméticas, manejar operaciones algebraicas, manejar identidades y razones trigonométricas, e identificar los lugares geométricos de rectas y cónicas. 


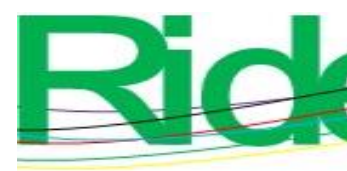

Revista Iberoamericana para la Investigación y el Desarrollo Educativo ISSN $2007-7467$

Debido a que el curso propedéutico dura solo 20 horas, se optó por cubrir el mínimo indispensable, es decir, lo básico de aritmética y álgebra. En reuniones de academia mediante trabajo colaborativo entre docentes, se seleccionaron los subtemas para aritmética: suma, resta, multiplicación y división de fracciones. En álgebra los subtemas fueron suma, resta, multiplicación y división de monomios, así como división de polinomios, potencias, raíces, binomio al cuadrado, binomio al cubo, factor común, factorización de un trinomio cuadrado perfecto, factorización de resta de cuadrados (conjugados), factorización de una resta o suma de cubos, factorización de la forma $\mathrm{ax}^{2}+\mathrm{bx}+\mathrm{c}$.

El diseño del instrumento fue elaborado por la Jefatura de División y Coordinación de Ciencias Básicas del TecMM, con el apoyo del docente Maestro Álvaro Sánchez Navarrete. La validación de los problemas o ítems del cuestionario fue realizado por el Departamento de División de Ciencias Básicas, la Coordinación y algunos docentes de matemáticas.

No se efectuó ninguna prueba piloto con alumnos, aunque el cuestionario fue aplicado a docentes de la academia para revisar posibles errores antes de su implementación. El cuestionario fue aplicado a los alumnos por parte de los docentes de la Academia de Ciencias Básicas, del 10 al 28 agosto de 2020 en la ciudad de Puerto Vallarta, Jalisco, México.

La administración del instrumento se hizo mediante un formulario de Google compartido a los alumnos en forma virtual a través de Whastapp, correo electrónico y un enlace en Classroom, esto por las medidas de confinamiento debido a la pandemia causada por el SARS-CoV-2. Este instrumento se aplicó en las seis carreras que tienen ingreso en el TecMM: ingeniería en Gestión Empresarial, ingenierías en Sistemas Computacionales, ingeniería en Electromecánica, Gastronomía, Arquitectura y Turismo. La evaluación previa y la posterior contenían los mismos ítems. La aplicación se programó para llevarse a cabo de forma asincrónica únicamente un día para cada evaluación. No se notificó a los estudiantes que se aplicaría el mismo instrumento al final, y como medida de seguridad no se mostraron resultados a los alumnos al finalizar las evaluaciones.

El curso propedéutico fue aplicado a todos los alumnos que efectuaron la evaluación al inicio y final. La muestra quedó conformada del siguiente modo: 25 alumnos de Arquitectura, 19 de Gastronomía, 20 de ingeniería en Electromecánica, 41 de ingeniería en Gestión Empresarial, 14 de ingeniería en Sistemas Computacionales y 15 de Turismo. A los docentes que impartieron el curso se les proporcionó una guía elaborada por profesores de la Academia de Ciencias Básicas. 

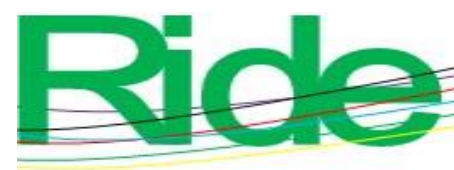

Revista Iberoamericana para la Investigación y el Desarrollo Educativo

ISSN $2007-7467$

Un elemento novedoso en el curso fue el uso de tecnología, es decir, las nuevas estrategias de aprendizaje y enseñanza aplicadas al curso, consistentes en videos elaborados por el docente con el software gratuito ScreenCast, así como por otros profesores, los cuales podían ser consultados en cualquier momento. Asimismo, se efectuaron videoconferencias para aclarar dudas o clases virtuales cuando el alumno lo necesitara, para lo cual se usaron pizarras electrónicas como Idroo, OneNote, etc.

Los datos obtenidos de la aplicación del instrumento se exportaron a una hoja de cálculo de Microsoft Office Excel 2007 para su procesamiento. Con la información de la base de datos se generaron tablas y gráficas que fueron analizadas. Finalmente, para sustentar las conclusiones se aplicó una prueba $\mathrm{Z}$ dependiente para muestras pareadas. De ese modo se compararon las diferencias entre los resultados previos y posteriores al curso remedial.

\section{Resultados}

En la tabla 1 se puede observar que en la carrera de ingeniería en Sistemas Computacionales se obtuvieron los resultados más altos después del curso propedéutico, con un aumento en el promedio de 25.1, seguido de Gastronomía con 12.6, Turismo con 11, Arquitectura con 10.4, ingeniería en Electromecánica con 10.3 e ingeniería en Gestión Empresarial con 0.2. Se aprecia que todas las carreras presentan diferencias significativas entre la evaluación previa y posterior. Esto señala que se mejoró el nivel académico de los alumnos en el área de matemáticas. 


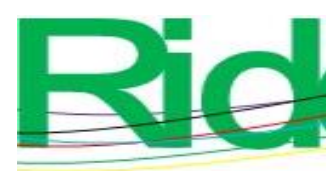

Revista Iberoamericana para la
Investigación y el Desarrollo Educativo
ISSN $2007-7467$

$\mu_{\mathrm{d}}$ es la media de las diferencias entre los promedios previos y posteriores al curso remedial. $H_{0}: \mu_{d}=0$

La hipótesis nula propuesta es que la diferencia de la media aritmética previa y posterior al curso de todos los alumnos es cero (es decir, nula). Esto significaría que no hubo cambio significativo; por lo tanto, el curso remedial de matemáticas no provocó ninguna mejora en las competencias de matemáticas de los alumnos.

$H_{1}: \mu_{d}>0$

La hipótesis alternativa planteada es que la diferencia de la media aritmética previa y posterior al curso de todos los alumnos es mayor a cero (es decir, hubo un aumento en el promedio), lo cual significaría que se obtuvo un cambio significativo. Esto representaría que el curso remedial de matemáticas sí provocó mejora en las competencias de matemáticas de los alumnos. Para $95 \%$ de confianza (es decir, un alfa de 0.5 ) se tiene un valor crítico de: $z_{\propto}=z_{0.05}=1.645$

Si el valor de $\mathrm{Z}$ calculado es mayor al valor crítico, decisión: $\mathrm{H}_{1}$ se acepta y $\mathrm{H}_{0}$ se rechaza.

Si el valor de $\mathrm{Z}$ calculado es menor al valor crítico, decisión: $\mathrm{H}_{1}$ se rechaza y $\mathrm{H}_{0}$ se acepta.

Se tiene una media aritmética de las diferencias igual a 11.6, una desviación estándar de 20.14 y un tamaño de muestra de 134 alumnos, por lo que el valor calculado para $\mathrm{Z}$ es de 6.67 .

$z=\frac{\bar{d}-d_{0}}{s_{d} / \sqrt{n}}$

$z=\frac{11.6}{20.14 / \sqrt{134}}=6.67$

En consecuencia, $\mathrm{H}_{1}$ se acepta y $\mathrm{H}_{0}$ se rechaza.

donde

$\mathrm{d}_{0}$ son las diferencias entre los promedios de la evaluación previos y posteriores al curso remedial que fueron calculados a cada uno de los 134 alumnos.

$\mathrm{S}_{\mathrm{d}}$ es la desviación estándar.

$\mathrm{n}$ es el tamaño de la muestra.

$\bar{d}$ es la media aritmética de las diferencias. 


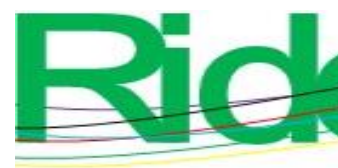

\section{Revista Iberoamericana para la Investigación y el Desarrollo Educativo ISSN $2007-7467$}

De acuerdo con el resultado de la prueba de hipótesis realizada, se puede afirmar con $95 \%$ de confianza que el curso propedéutico incide positivamente en mejorar las competencias de matemáticas en los alumnos de nuevo ingreso.

En la tabla 2 se observa que previo al curso propedéutico, la calificación con mayor frecuencia era 75, y posterior al curso fue de 100. Esto indica una mejora en las respuestas a los problemas de matemáticas planteados luego de recibir el curso propedéutico $\mathrm{y}$, en consecuencia, una mejora en el nivel académico. La mediana previa al curso fue de 70, y la posterior fue de 81 , lo que termina confirmando lo mismo que la moda.

Tabla 2. Otros estadísticos

\begin{tabular}{|c|c|c|}
\hline Otros estadísticos & Previo & Posterior \\
\hline Moda & 75 & 100 \\
\hline Mediana & 70 & 81 \\
\hline
\end{tabular}

Fuente: Elaboración propia

En la figura 1 se observa el diagrama de dispersión de las diferencias de los promedios previos y posteriores al curso propedéutico de los 134 alumnos. Si la diferencia es positiva, significa que el promedio del alumno mejoró después del curso propedéutico. La mayoría de las diferencias son superiores a cero (es decir, positivas), lo que significa un resultado favorable al implementar dicho curso. 
Figura 1. Diagrama de dispersión de las diferencias

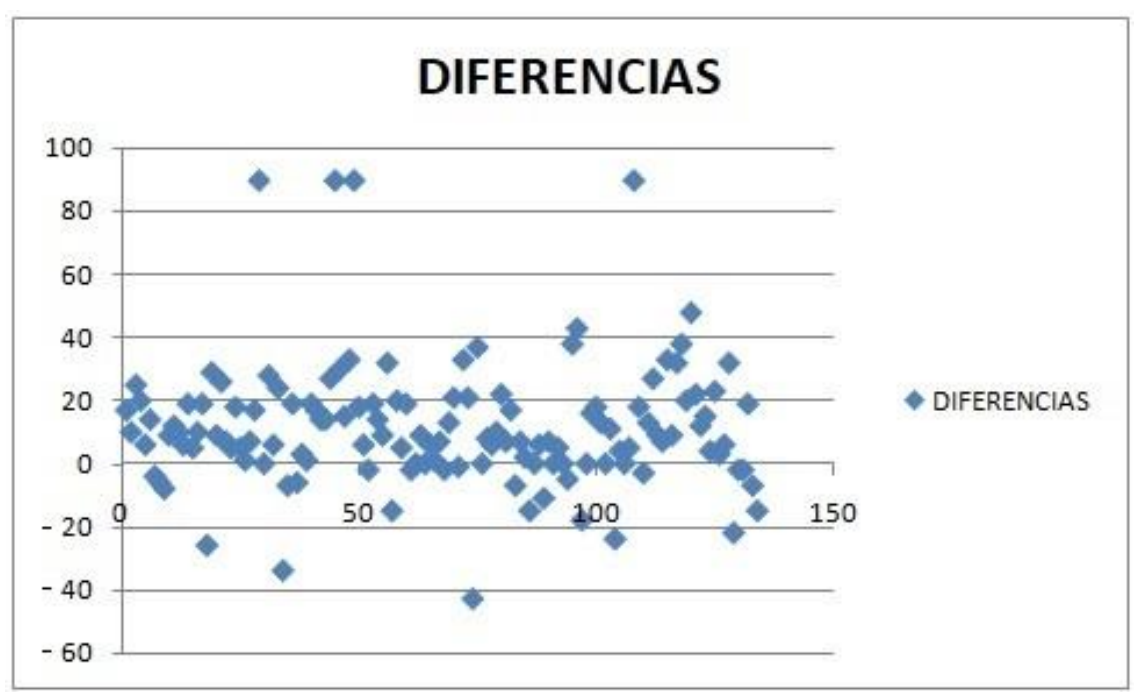

Fuente: Elaboración propia

La figura 2 contiene los promedios antes y después del curso propedéutico. Se identifica una mejoría posterior al curso propedéutico impartido en todas las carreras. La carrera que presenta la mejoría más notable fue Sistemas Computacionales, y la carrera donde dicha diferencia no fue tan significativa fue en Ingeniería en Gestión Empresarial.

Figura 2. Gráfica de diferencia de promedios por carrera antes y después del curso propedéutico

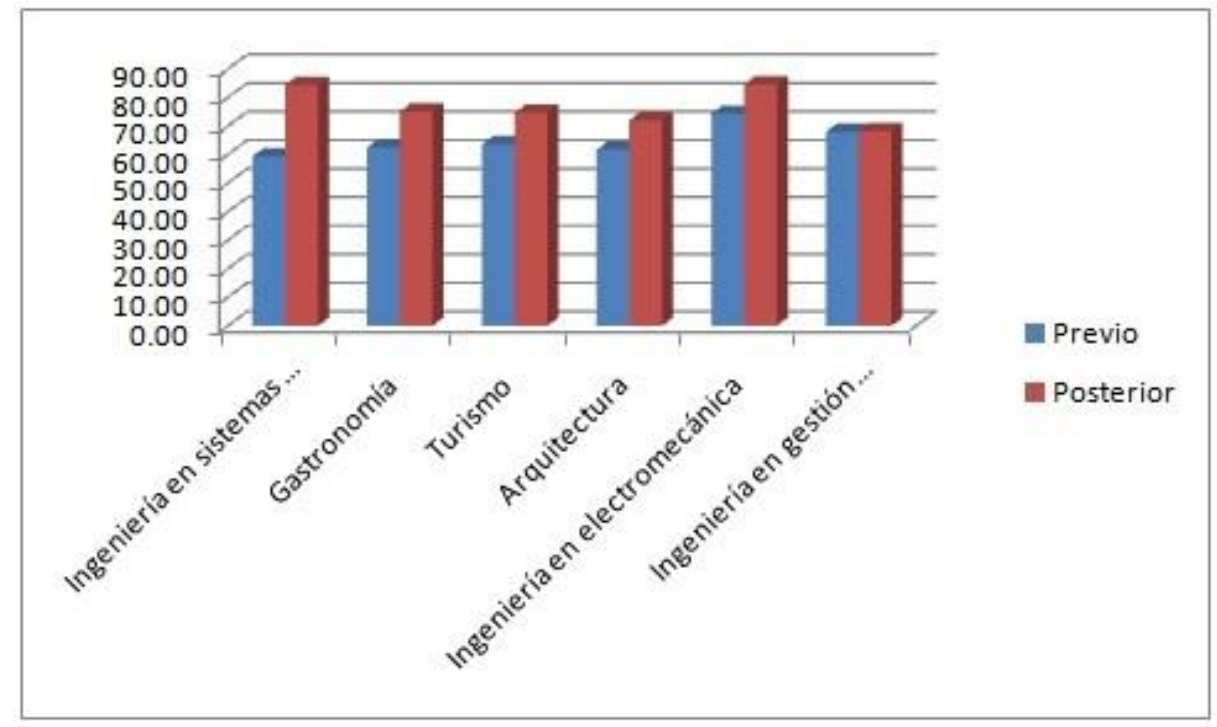

Fuente: Elaboración propia 


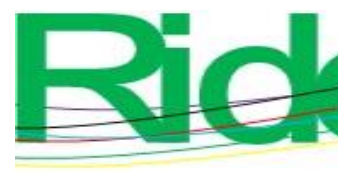

운

Revista Iberoamericana para la

Investigación y el Desarrollo Educativo

ISSN $2007-7467$

La tabla 3 muestra la cantidad de alumnos aprobados antes (69) y después (95) del curso propedéutico. Asimismo, el porcentaje de aprobados previo y posterior al curso fueron $51.49 \%$ y $70.9 \%$, respectivamente. Para esto, se consideró que la calificación mínima aprobatoria en la institución donde se realizó la investigación era de 70. El aumento en el porcentaje de alumnos aprobados fue cercano a $20 \%$, lo que da cuenta de un incremento significativo en las calificaciones previas y posteriores al curso.

Tabla 3. Alumnos aprobados

\begin{tabular}{|c|c|}
\hline Previo & Posterior \\
\hline 69 alumnos & 95 alumnos \\
\hline $51.49 \%$ & $70.9 \%$ \\
\hline
\end{tabular}

Fuente: Elaboración propia

\section{Discusión}

Vaca Haro y Estévez (2017) identificaron la importancia del desarrollo del pensamiento lógico formal (operaciones formales de las etapas de Piaget) como un criterio para desarrollar aprendizajes significativos en estudiantes universitarios. Estos autores argumentan que los alumnos que solo alcanzan a desarrollar etapas previas en su desarrollo mental tienen dificultades en su rendimiento académico en matemáticas, lo cual es congruente con la problemática planteada en este estudio, donde se identificó una gran cantidad de alumnos con bajo rendimiento documentado en las pruebas PLANEA que puede estar relacionado con un bajo nivel de desarrollo cognitivo y con dificultades para cursar los estudios universitarios, particularmente en el área de matemáticas.

Por otra parte, Alting y Walser (2006) encontraron que la buena preparación en matemáticas de estudiantes universitarios de nuevo ingreso es un buen predictor de la permanencia en la institución. También Ennis et al. (2013) identificaron una relación entre los altos puntajes en matemáticas en preparatoria y el cursar de forma exitosa la materia Cálculo I en la universidad.

Además, los bajos niveles de rendimiento en estudiantes de nuevo ingreso a las universidades han sido documentados en diversos estudios y contextos (Aparicio, 2006; 


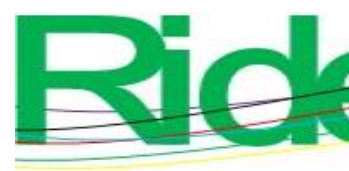

Revista Iberoamericana para la
Investigación y el Desarrollo Educativo
ISSN $2007-7467$

Ennis et al., 2013; Gaete y Jiménez, 2011; Martínez et al., 1998; Petriz et al., 2010; Posso, 2005; Zámková et al., 2016), por lo que resulta relevante diseñar estrategias que contribuyan a mejorar las competencias en matemáticas de estudiantes de nuevo ingreso.

Por otra parte, Aparicio (2006) describió una problemática de permanencia y eficiencia terminal en la Universidad Autónoma de Yucatán relacionada con deficiencias y rezago en las materias de álgebra y cálculo. Este autor explica que estrategias como cursos propedéuticos o talleres extracurriculares no habían generado los resultados deseados en los indicadores institucionales. Aun así, la importancia del presente estudio radica en se procuró probar la efectividad del curso propedéutico analizando, primeramente, su efecto en el aprendizaje (comparación de las evaluaciones inicial y final), y posteriormente verificando si esta estrategia tendría un impacto positivo en la reducción de los índices de reprobación y deserción.

En estudios similares se han planteado diversas estrategias para mejorar el nivel de competencias matemáticas de los estudiantes de nuevo ingreso a la educación superior. Por ejemplo, Zámková et al. (2016) formularon un examen diagnóstico en el College of Polytechniques Jihlava (República Checa) para determinar cuáles alumnos se pueden inscribir a los cursos de matemáticas regulares y cuáles requieren de un curso previo (seminario de matemáticas) para reforzar los aprendizajes del nivel de preparatoria. Esta metodología, de acuerdo con sus autores, ha generado resultados favorables en la disminución de los índices de reprobación, aunque valga acotar que se diferencia de la ofrecida en el presente trabajo porque no se aplicó a todos los alumnos de nuevo ingreso, sino a unos elegidos previamente.

Otra estrategia innovadora es la implementada en la University of Colorado Boulder, consistente en un sistema web basado en inteligencia artificial para el aprendizaje de matemáticas con dos funciones: una función para desarrollar competencias de matemáticas y la otra de diagnóstico para identificar los niveles de desarrollo de los estudiantes y predecir sus posibilidades de éxito en diferentes cursos. Esto sirve como referencia para planificar una ruta de aprendizaje para los estudiantes y para determinar quiénes requieren de cursos previos en las materias de los programas de estudio (Ennis et al., 2013).

Por su parte, Yánez y Nevárez (2018) utilizaron el software libre eXe-Learning en la Pontificia Universidad Católica de Ecuador con los alumnos de nuevo ingreso como una estrategia complementaria para facilitar el aprendizaje. Los autores concluyen que la 


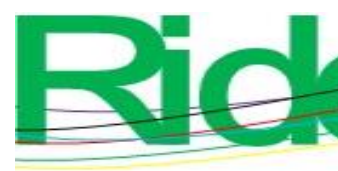

Revista Iberoamericana para la Investigación y el Desarrollo Educativo ISSN $2007-7467$

didáctica de la enseñanza apoyada con dicha herramienta contribuye a mejorar el desempeño académico de los alumnos.

En síntesis, los estudios mencionados dan cuenta de opciones que tienen un impacto favorable para nivelar a los estudiantes de nuevo ingreso de educación superior respecto a los aprendizajes esperados de educación media superior. De hecho, se documentaron dos estudios muy similares a este: uno donde se señala que no hubo resultados positivos en un curso propedéutico (Aparicio, 2006) y otro donde un curso previo a las materias regulares ha tenido un impacto positivo en los indicadores institucionales (Zámková et al., 2016).

En definitiva, los resultados iniciales del curso propedéutico presentado en este estudio son favorables en cuanto a la nivelación de competencias matemáticas. Esto es una fortaleza de la investigación, la cual puede considerarse como referencia para el diseño de estrategias similares; sin embargo, como una limitación queda pendiente analizar si los aprendizajes obtenidos tendrán también un impacto favorable en los indicadores de reprobación y deserción, con resultados tangibles a largo plazo. Para ello, deberá hacerse el seguimiento académico de los alumnos que participaron en este estudio.

\section{Conclusiones}

Los altos índices de reprobación y deserción como consecuencia del rezago académico en el área de matemáticas demandan el diseño de estrategias para mejorar el nivel académico, así como para disminuir estos estadísticos (reprobación y deserción). Una de estas estrategias es el curso propedéutico que el TecMM realiza concentrando temas de aritmética y álgebra, los cuales son evaluados a través de una prueba previa y posterior considerando las competencias esperadas de nivel medio superior y los prerrequisitos de las materias de licenciatura. En este estudio se logró concluir — mediante la aplicación del estadístico prueba $\mathrm{Z}$ dependiente para muestras pareadas para comparar la evaluación inicial y final- que "el curso incidió en mejorar las deficiencias previas de conocimientos matemáticos de forma significativa en alumnos de nuevo ingreso del TecMM con un nivel de confianza de $95 \%$ \%. En consecuencia, dicha estrategia se considera valiosa y útil, y puede llegar a incidir de forma favorable en los indicadores institucionales de reprobación, deserción y eficiencia terminal, aunque para poder comprobarlo se requiere hacer un seguimiento para determinar el impacto del curso remedial en estos indicadores. 


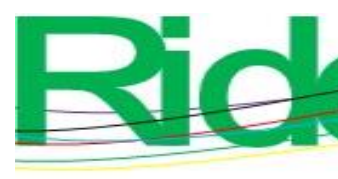
Revista Iberoamericana para la
Investigación y el Desarrollo Educativo
ISSN $2007-7467$

\section{Futuras líneas de investigación}

En esta investigación se realizó una propuesta para combatir la reprobación en matemáticas de nivel superior. Sin embargo, en futuras investigaciones se debe profundizar en las causas de la reprobación y deserción en instituciones de nivel superior, así como en las estrategias de aprendizaje y enseñanza utilizadas para establecer si se debe continuar con ellas o si deben ser cambiadas o modificadas. También se debe revisar en el futuro el resultado de las asignaturas de matemáticas que cursarán los estudiantes a los cuales fue aplicada esta estrategia para observar con más detalle el impacto logrado.

\section{Referencias}

Alting, A. and Walser, A. (2006). The Influence Of Mathematics Preparation On The Retention And Academic Achievement Of Underrepresented Engineering Students. In Proceedings of the 2006 American Society for Engineering Educations Annual Conference and Exposition. 2006 Annual Conference \& Exposition. Chicago, Illinois: ASEE. Retrieved from https://peer.asee.org/the-influence-of-mathematicspreparation-on-the-retention-and-academic-achievement-of-underrepresentedengineering-students

Aparicio, E. (2006). Un estudio sobre factores que obstaculizan la permanencia, logro educativo y eficiencia terminal en las áreas de matemáticas del nivel superior: el caso de la Facultad de Matemáticas de la Universidad Autónoma de Yucatán. Acta Latinoamericana de Matemática Educativa, 19, 450-455.

Castro, F., Piñon, A. y Avilés, J. (2015). La experiencia de un curso de fortalecimiento académico en el bachillerato universitario para facilitar el ingreso y permanencia a la educación superior: logros y desafíos. Congreso CLABES. Recuperado de https://revistas.utp.ac.pa/index.php/clabes/article/view/1093

Ennis, T. D., Sullivan, J. F., Louie, B. and Knight, D. (2013). Unlocking the gate to calculus success: Pre-calculus for engineers an assertive approach to readying underprepared students. In Proceedings of the 2013 American Society for Engineering Educations Annual Conference and Exposition. Atlanta GA. Retrieved from https://peer.asee.org/unlocking-the-gate-to-calculus-success-pre-calculus-forengineers-an-assertive-approach-to-readying-underprepared-students 


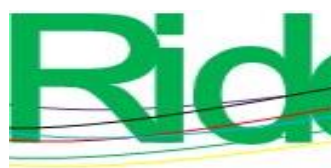

Revista Iberoamericana para la Investigación y el Desarrollo Educativo ISSN 2007 - 7467

Gaete, M. y Jiménez W. (2011). Carencias en la formación inicial y continua de los docentes y bajo rendimiento escolar en matemática en Costa Rica. Cuadernos de Investigación y Formación en Educación Matemática, 6(9), 93-117.

García-Oliveros, G., Salguero-Rivera, B., Rodríguez-Díaz, O., Palomino-Bejarano, E., y Caicedo-Valencia, R. (2020). Las prácticas de evaluación de las matemáticas universitarias: tensiones y desafíos desde la red conceptual en la que se inscriben. Uniciencia, 34(1), 246-262.

Gil, S. B. y Pérez, C. E. (2013). Estrategias desarrolladas en el Cecyt 171 del IPN campus León Guanajuato, a fin de reducir aprendizajes pobres en álgebra disminuyendo el abandono escolar. Congreso CLABES. Recuperado de https://revistas.utp.ac.pa/index.php/clabes/article/view/976

Martínez, M. Vivaldo, J., Navarro, M., González, M. y Jerónimo, J. (1998). Análisis multirreferencial del fenómeno de la reprobación en estudiantes universitarios mexicanos. Psicología Escolar e Educacional, 2(2), 161-174.

Petriz Mayen, M. A., Barona Ríos, C., López Villareal, R. M. y Quiroz González, J. (2010). Niveles de desempeño y actitudes hacia las matemáticas en estudiantes de la licenciatura en administración en una universidad estatal mexicana. Revista Mexicana de Investigación Educativa, 15(47), 1223-1249.

Posso, A. (2005). Sobre el bajo aprovechamiento en el curso de matemáticas I de la UTP. Scientia Et Technica, 11(28), 169-174.

Romero-Bojórquez, L., Utrilla-Quiroz, A., y Utrilla-Quiroz, V. M. (2014). Las actitudes positivas y negativas de los estudiantes en el aprendizaje de las matemáticas: su impacto en la reprobación y la eficiencia terminal. Ra Ximhai, 10(5), 291-319.

Secretaría de Educación Pública (SEP) (2017). Educación media superior: bases de datos 2017. Recuperado de http://planea.sep.gob.mx/ms/base_de_datos_2017/

Shena, C., Mielea, D. and Vasilyeva, M. (2016). The relation between college students' academic mindsets and their persistence during math problema solving. Psychology in Russia: State of the Art, 9(3), 1-19.

Vaca Haro, A. y Estévez, E. (2017). Relación entre el rendimiento académico en matemática y el nivel operativo piagetiano. Revista Publicando, 4(11-1), 287-301. Recuperado de https://revistapublicando.org/revista/index.php/crv/article/view/525

Vargas, M. y Montero, E. (2016). Factores que determinan el rendimiento académico en matemáticas en el contexto de una universidad tecnológica: aplicación de un modelo 
de ecuaciones estructurales. Universitas Psychologica, 15(4), 1-11. Recuperado de https://dialnet.unirioja.es/servlet/articulo?codigo $=6703743$

Vila, R. y Rubio, M. (2016). Actitudes hacia la estadística en el alumnado del grado de pedagogía de la Universidad de Barcelona. REDU. Revista de Docencia Universitaria, 14(1), 131-149.

Yánez, V. y Nevárez, M. (2018). Exelearning: recurso digital de una estrategia didáctica de enseñanza-aprendizaje de matemática. 3C TIC. Cuadernos de desarrollo aplicados a las TIC, 7(4), 98-121. Recuperado de https://ojs.3ciencias.com/index.php/3ctic/article/view/717

Zámková, M., Prokop, M. and Stolín, R. (2016). Development of the success rate in mathematics 1 at the college of polytechnics Jihlava (Czech Republic) in 2006-2015. The Turkish Online Journal of Educational Technology, Special Issue for INTE. 700708. Retrieved from https://starfos.tacr.cz/en/result/RIV\%2F71226401\%3A $\% 2 \mathrm{~F} 16 \% 3 \mathrm{AN} 0000040$ 


\begin{tabular}{|c|c|}
\hline$\longrightarrow A$ & $\begin{array}{l}\text { Revista Iberoamericana para la } \\
\text { Investigación y el Desarrollo Educativo } \\
\text { ISSN } 2007-7467\end{array}$ \\
\hline Rol de Contribución & Autor (es) \\
\hline Conceptualización & María Luisa Torres Isiordia \\
\hline Metodología & Carlos Miguel Amador Ortíz \\
\hline Software & No aplica \\
\hline Validación & Leticia Velarde Peña \\
\hline Análisis Formal & María Luisa Torres Isiordia \\
\hline Investigación & $\begin{array}{l}\text { Carlos Miguel Amador Ortíz (principal) } \\
\text { Leticia Velarde Peña (apoyo) } \\
\text { María Luisa Torres Isiordia (apoyo) }\end{array}$ \\
\hline Recursos & $\begin{array}{l}\text { Carlos Miguel Amador Ortíz (principal) } \\
\text { Leticia Velarde Peña (apoyo) } \\
\text { María Luisa Torres Isiordia (apoyo) }\end{array}$ \\
\hline Curación de datos & $\begin{array}{l}\text { Carlos Miguel Amador Ortíz (principal) } \\
\text { Leticia Velarde Peña (apoyo) } \\
\text { María Luisa Torres Isiordia (apoyo) }\end{array}$ \\
\hline $\begin{array}{l}\text { Escritura - Preparación del } \\
\text { borrador original }\end{array}$ & María Luisa Torres Isiordia \\
\hline $\begin{array}{l}\text { Escritura - Revisión y } \\
\text { edición }\end{array}$ & Carlos Miguel Amador Ortíz \\
\hline Visualización & María Luisa Torres Isiordia \\
\hline Supervisión & Carlos Miguel Amador Ortíz \\
\hline Administración de Proyectos & María Luisa Torres Isiordia \\
\hline Adquisición de fondos & No aplica \\
\hline
\end{tabular}

